\title{
Kolmogorov's Zero-One Law
}

\author{
Agnes Doll \\ Ludwig Maximilian University of Munich \\ Germany
}

\begin{abstract}
Summary. This article presents the proof of Kolmogorov's zero-one law in probability theory. The independence of a family of $\sigma$-fields is defined and basic theorems on it are given.
\end{abstract}

MML identifier: KOLMOG01, version: $\underline{7.11 .01 \quad 4.117 .1046}$

The articles [8], [19], [2], [10], [12], [18], [20], [1], [15], [5], [21], [11], [3], [9], [7], $[6],[17],[4],[16],[14]$, and [13] provide the terminology and notation for this paper.

For simplicity, we adopt the following convention: $\Omega, I$ are non empty sets, $\mathcal{F}$ is a $\sigma$-field of subsets of $\Omega, P$ is a probability on $\mathcal{F}, D, E, F$ are families of subsets of $\Omega, A, B, s$ are non empty subsets of $\mathcal{F}, b$ is an element of $B, a$ is an element of $\mathcal{F}, p, q, u, v$ are events of $\mathcal{F}, n$ is an element of $\mathbb{N}$, and $i$ is a set.

Next we state three propositions:

(1) For every function $f$ and for every set $X$ such that $X \subseteq \operatorname{dom} f$ holds if $X \neq \emptyset$, then $\operatorname{rng}(f\lceil X) \neq \emptyset$.

(2) For every real number $r$ such that $r \cdot r=r$ holds $r=0$ or $r=1$.

(3) For every family $X$ of subsets of $\Omega$ such that $X=\emptyset$ holds $\sigma(X)=\{\emptyset, \Omega\}$.

Let $\Omega$ be a non empty set, let $\mathcal{F}$ be a $\sigma$-field of subsets of $\Omega$, let $B$ be a subset of $\mathcal{F}$, and let $P$ be a probability on $\mathcal{F}$. The functor $\operatorname{Indep}(B, P)$ yielding a subset of $\mathcal{F}$ is defined as follows:

(Def. 1) For every element $a$ of $\mathcal{F}$ holds $a \in \operatorname{Indep}(B, P)$ iff for every element $b$ of $B$ holds $P(a \cap b)=P(a) \cdot P(b)$.

Next we state several propositions:

(4) Let $f$ be a sequence of subsets of $\mathcal{F}$. Suppose for all $n, b$ holds $P(f(n) \cap$ $b)=P(f(n)) \cdot P(b)$ and $f$ is disjoint valued. Then $P(b \cap \bigcup f)=P(b)$. $P(\bigcup f)$. 
(5) $\operatorname{Indep}(B, P)$ is a Dynkin system of $\Omega$.

(6) For every family $A$ of subsets of $\Omega$ such that $A$ is intersection stable and $A \subseteq \operatorname{Indep}(B, P)$ holds $\sigma(A) \subseteq \operatorname{Indep}(B, P)$.

(7) Let $A, B$ be non empty subsets of $\mathcal{F}$. Then $A \subseteq \operatorname{Indep}(B, P)$ if and only if for all $p, q$ such that $p \in A$ and $q \in B$ holds $p$ and $q$ are independent w.r.t. $P$.

(8) For all non empty subsets $A, B$ of $\mathcal{F}$ such that $A \subseteq \operatorname{Indep}(B, P)$ holds $B \subseteq \operatorname{Indep}(A, P)$.

(9) Let $A$ be a family of subsets of $\Omega$. Suppose $A$ is a non empty subset of $\mathcal{F}$ and intersection stable. Let $B$ be a non empty subset of $\mathcal{F}$. Suppose $B$ is intersection stable. If $A \subseteq \operatorname{Indep}(B, P)$, then for all $D, s$ such that $D=B$ and $\sigma(D)=s$ holds $\sigma(A) \subseteq \operatorname{Indep}(s, P)$.

(10) Let given $E, F$. Suppose that

(i) $E$ is a non empty subset of $\mathcal{F}$ and intersection stable, and

(ii) $\quad F$ is a non empty subset of $\mathcal{F}$ and intersection stable.

Suppose that for all $p, q$ such that $p \in E$ and $q \in F$ holds $p$ and $q$ are independent w.r.t. P. Let given $u$, $v$. If $u \in \sigma(E)$ and $v \in \sigma(F)$, then $u$ and $v$ are independent w.r.t. $P$.

Let $I$ be a set, let $\Omega$ be a non empty set, and let $\mathcal{F}$ be a $\sigma$-field of subsets of $\Omega$. A function from $I$ into $2^{\mathcal{F}}$ is said to be a many sorted $\sigma$-field over $I$ and $\mathcal{F}$ if:

(Def. 2) For every $i$ such that $i \in I$ holds it $(i)$ is a $\sigma$-field of subsets of $\Omega$.

Let $\Omega$ be a non empty set, let $\mathcal{F}$ be a $\sigma$-field of subsets of $\Omega$, let $P$ be a probability on $\mathcal{F}$, let $I$ be a set, and let $A$ be a function from $I$ into $\mathcal{F}$. We say that $A$ is independent w.r.t. $P$ if and only if:

(Def. 3) For every one-to-one finite sequence $e$ of elements of $I$ such that $e \neq \emptyset$ holds $\prod(P \cdot A \cdot e)=P(\bigcap \operatorname{rng}(A \cdot e))$.

Let $\Omega$ be a non empty set, let $\mathcal{F}$ be a $\sigma$-field of subsets of $\Omega$, let $I$ be a set, let $J$ be a subset of $I$, and let $F$ be a many sorted $\sigma$-field over $I$ and $\mathcal{F}$. A function from $J$ into $\mathcal{F}$ is said to be a $\sigma$-section over $J$ and $F$ if:

(Def. 4) For every $i$ such that $i \in J$ holds it $(i) \in F(i)$.

Let $\Omega$ be a non empty set, let $\mathcal{F}$ be a $\sigma$-field of subsets of $\Omega$, let $P$ be a probability on $\mathcal{F}$, let $I$ be a set, and let $F$ be a many sorted $\sigma$-field over $I$ and $\mathcal{F}$. We say that $F$ is independent w.r.t. $P$ if and only if:

(Def. 5) For every finite subset $E$ of $I$ holds every $\sigma$-section over $E$ and $F$ is independent w.r.t. $P$.

Let $I$ be a set, let $\Omega$ be a non empty set, let $\mathcal{F}$ be a $\sigma$-field of subsets of $\Omega$, let $F$ be a many sorted $\sigma$-field over $I$ and $\mathcal{F}$, and let $J$ be a subset of $I$. Then $F\left\lceil J\right.$ is a function from $J$ into $2^{\mathcal{F}}$. 
Let $I$ be a set, let $J$ be a subset of $I$, let $\Omega$ be a non empty set, let $\mathcal{F}$ be a $\sigma$-field of subsets of $\Omega$, and let $F$ be a function from $J$ into $2^{\mathcal{F}}$. Then $\bigcup F$ is a family of subsets of $\Omega$.

Let $I$ be a set, let $\Omega$ be a non empty set, let $\mathcal{F}$ be a $\sigma$-field of subsets of $\Omega$, let $F$ be a many sorted $\sigma$-field over $I$ and $\mathcal{F}$, and let $J$ be a subset of $I$. The functor $\operatorname{sig} \operatorname{Un}(F, J)$ yields a $\sigma$-field of subsets of $\Omega$ and is defined as follows:

(Def. 6) $\operatorname{sigUn}(F, J)=\sigma(\bigcup(F \nmid J))$.

Let $I$ be a set, let $\Omega$ be a non empty set, let $\mathcal{F}$ be a $\sigma$-field of subsets of $\Omega$, and let $F$ be a many sorted $\sigma$-field over $I$ and $\mathcal{F}$. The functor futSigmaFields $(F, I)$ yielding a family of subsets of $2^{\Omega}$ is defined as follows:

(Def. 7) For every family $S$ of subsets of $\Omega$ holds $S \in \operatorname{futSigmaFields}(F, I)$ iff there exists a finite subset $E$ of $I$ such that $S=\operatorname{sigUn}(F, I \backslash E)$.

Let $I$ be a set, let $\Omega$ be a non empty set, let $\mathcal{F}$ be a $\sigma$-field of subsets of $\Omega$, and let $F$ be a many sorted $\sigma$-field over $I$ and $\mathcal{F}$. Note that futSigmaFields $(F, I)$ is non empty.

Let $I$ be a set, let $\Omega$ be a non empty set, let $\mathcal{F}$ be a $\sigma$-field of subsets of $\Omega$, and let $F$ be a many sorted $\sigma$-field over $I$ and $\mathcal{F}$. The functor tailSigmaField $(F, I)$ yielding a family of subsets of $\Omega$ is defined as follows:

(Def. 8) tailSigmaField $(F, I)=\bigcap$ futSigmaFields $(F, I)$.

Let $I$ be a set, let $\Omega$ be a non empty set, let $\mathcal{F}$ be a $\sigma$-field of subsets of $\Omega$, and let $F$ be a many sorted $\sigma$-field over $I$ and $\mathcal{F}$. Note that tailSigmaField $(F, I)$ is non empty.

Let $\Omega$ be a non empty set, let $\mathcal{F}$ be a $\sigma$-field of subsets of $\Omega$, let $I$ be a non empty set, let $J$ be a non empty subset of $I$, and let $F$ be a many sorted $\sigma$-field over $I$ and $\mathcal{F}$. The functor MeetSections $(J, F)$ yields a family of subsets of $\Omega$ and is defined by the condition (Def. 9).

(Def. 9) Let $x$ be a subset of $\Omega$. Then $x \in \operatorname{MeetSections}(J, F)$ if and only if there exists a non empty finite subset $E$ of $I$ and there exists a $\sigma$-section $f$ over $E$ and $F$ such that $E \subseteq J$ and $x=\bigcap \operatorname{rng} f$.

One can prove the following propositions:

(11) For every many sorted $\sigma$-field $F$ over $I$ and $\mathcal{F}$ and for every non empty subset $J$ of $I$ holds $\sigma(\operatorname{MeetSections}(J, F))=\operatorname{sigUn}(F, J)$.

(12) Let $F$ be a many sorted $\sigma$-field over $I$ and $\mathcal{F}$ and $J, K$ be non empty subsets of $I$. Suppose $F$ is independent w.r.t. $P$ and $J$ misses $K$. Let $a$, $c$ be subsets of $\Omega$. If $a \in \operatorname{MeetSections}(J, F)$ and $c \in \operatorname{MeetSections}(K, F)$, then $P(a \cap c)=P(a) \cdot P(c)$.

(13) Let $F$ be a many sorted $\sigma$-field over $I$ and $\mathcal{F}$ and $J$ be a non empty subset of $I$. Then MeetSections $(J, F)$ is a non empty subset of $\mathcal{F}$.

Let us consider $I, \Omega, \mathcal{F}$, let $F$ be a many sorted $\sigma$-field over $I$ and $\mathcal{F}$, and let $J$ be a non empty subset of $I$. Observe that MeetSections $(J, F)$ is intersection 
stable.

The following proposition is true

(14) Let $F$ be a many sorted $\sigma$-field over $I$ and $\mathcal{F}$ and $J, K$ be non empty subsets of $I$. Suppose $F$ is independent w.r.t. $P$ and $J$ misses $K$. Let given $u, v$. If $u \in \operatorname{sigUn}(F, J)$ and $v \in \operatorname{sigUn}(F, K)$, then $P(u \cap v)=P(u) \cdot P(v)$.

Let $I$ be a set, let $\Omega$ be a non empty set, let $\mathcal{F}$ be a $\sigma$-field of subsets of $\Omega$, and let $F$ be a many sorted $\sigma$-field over $I$ and $\mathcal{F}$. The functor finSigmaFields $(F, I)$ yielding a family of subsets of $\Omega$ is defined as follows:

(Def. 10) For every subset $S$ of $\Omega$ holds $S \in$ finSigmaFields $(F, I)$ iff there exists a finite subset $E$ of $I$ such that $S \in \operatorname{sigUn}(F, E)$.

One can prove the following propositions:

(15) For every many sorted $\sigma$-field $F$ over $I$ and $\mathcal{F}$ holds tail $\operatorname{SigmaField}(F, I)$ is a $\sigma$-field of subsets of $\Omega$.

(16) Let $F$ be a many sorted $\sigma$-field over $I$ and $\mathcal{F}$. If $F$ is independent w.r.t. $P$ and $a \in \operatorname{tailSigmaField}(F, I)$, then $P(a)=0$ or $P(a)=1$.

\section{ACKNOWLEDGMENTS}

The author wishes to express her sincere appreciation to Prof. F. Merkl for his kind support and encouragement during the course of this work.

\section{REFERENCES}

[1] Grzegorz Bancerek. König's theorem. Formalized Mathematics, 1(3):589-593, 1990.

[2] Grzegorz Bancerek. The ordinal numbers. Formalized Mathematics, 1(1):91-96, 1990.

[3] Grzegorz Bancerek and Krzysztof Hryniewiecki. Segments of natural numbers and finite sequences. Formalized Mathematics, 1(1):107-114, 1990.

[4] Czesław Byliński. Binary operations applied to finite sequences. Formalized Mathematics, 1(4):643-649, 1990.

[5] Czesław Byliński. Functions and their basic properties. Formalized Mathematics, 1(1):5565, 1990.

[6] Czesław Byliński. Functions from a set to a set. Formalized Mathematics, 1(1):153-164, 1990.

[7] Czesław Byliński. Partial functions. Formalized Mathematics, 1(2):357-367, 1990.

[8] Czesław Byliński. Some basic properties of sets. Formalized Mathematics, 1(1):47-53, 1990.

[9] Czesław Byliński. The sum and product of finite sequences of real numbers. Formalized Mathematics, 1(4):661-668, 1990.

[10] Agata Darmochwał. Finite sets. Formalized Mathematics, 1(1):165-167, 1990.

[11] Jarosław Kotowicz. Real sequences and basic operations on them. Formalized Mathematics, 1(2):269-272, 1990.

[12] Franz Merkl. Dynkin's lemma in measure theory. Formalized Mathematics, 9(3):591-595, 2001.

[13] Andrzej Nędzusiak. Probability. Formalized Mathematics, 1(4):745-749, 1990.

[14] Andrzej Nędzusiak. $\sigma$-fields and probability. Formalized Mathematics, 1(2):401-407, 1990.

[15] Beata Padlewska. Families of sets. Formalized Mathematics, 1(1):147-152, 1990.

[16] Alexander Yu. Shibakov and Andrzej Trybulec. The Cantor set. Formalized Mathematics, 5(2):233-236, 1996.

[17] Andrzej Trybulec. Binary operations applied to functions. Formalized Mathematics, 1(2):329-334, 1990. 
[18] Andrzej Trybulec and Agata Darmochwał. Boolean domains. Formalized Mathematics, 1(1):187-190, 1990.

[19] Zinaida Trybulec. Properties of subsets. Formalized Mathematics, 1(1):67-71, 1990.

[20] Edmund Woronowicz. Relations and their basic properties. Formalized Mathematics, 1(1):73-83, 1990.

[21] Edmund Woronowicz. Relations defined on sets. Formalized Mathematics, 1(1):181-186, 1990.

Received November 4, 2008 